\title{
Developments of Omni-directional Mobile Systems with Intuitive Operation for Care-Robots
}

\author{
Asuka Abe ${ }^{\mathrm{a}}$, Kazuki Tamura ${ }^{\mathrm{a}, \mathrm{b}}$, Yohei Tanaka ${ }^{\mathrm{a}}$, Arata Yasuda ${ }^{\mathrm{a}}$, Michiaki Shishido ${ }^{\mathrm{a}, *}$ \\ a National Institute of Technology, Tsuruoka College, Sawada104, Inooka,Tsuruoka,997-8511, Japan \\ ${ }^{\mathrm{b}}$ Chiba University, Yayoimachi 1-33, Yoyoicho, Inageku, Chiba, 263-8522, Japan \\ *Corresponding Author: m-shishido@tsuruoka-nct.ac.jp
}

\begin{abstract}
This study proposed an unique intuitive movement system which is equipped Omni-wheels with load cells for care-robots. In this systems, pilot or subjects can control intuitively with difference of centroid position with load cells. This systems is constructed a cylinder structure stacking a disk on a triangular frame and installed 3 load cells and Omni-wheels. These load cells sensed position and centroid is calculated by microcomputer. This sensed centroid position is applied for calculating direction and speed and these information transferred to servo systems installed Omni-wheels. The system was investigated with transient response characteristics and error distance of centroid of the system. Transient response measurement of the servo system were under no-load or $60 \mathrm{~kg}$ loaded condition respectively. the system were observed possible to move under $60 \mathrm{~kg}$ load. Moreover, suitable rotating speed of the servo system range is cleared from 2540 to 6349 rpm by responding time and stable rotating speed. Via the measuring error distance of centroid under loads, the maximum error distance was just $49 \mathrm{~mm}$ under heavy load. Therefore, this systems was possible to operate accurately under heavy load like $60 \mathrm{~kg}$ human. This Omni-directional mobile systems with intuitive operation is expect possible to be expected, novel and unique as novel care-robots technology.
\end{abstract}

Keywords: Care-robots, Intuitive operation, Omni-wheels.

\section{Introduction}

In recent Japanese society, lack of human resource of nursing care is serious and important problem. On the other hands, number of aging family-carers are increasing because decreasing birthrate and aging population in Japan.
(1) Therefore, the workloads of carers is huger and more serious topic in Japanese society.

On the basis of such as social background, to care these care-receivers like elder or handicap persons, applied robotic technology for nursing care is expected. ${ }^{(2)}$ However, in current society, such technology is not enough to be applied for real care-work scene. Because the operating these care-robots is too complicated to control and manage them. Sometimes carers have to learn and train long time to manipulate and control many parts and functions of robots. Especially, it is difficult for carers worked at real care-work scene to feedback to robots providers. However, according to increasing population of care-receiver in near future society, also care workers will be required more and more. Therefore, developing nursing care robots with simple interface and intuitive operation is required for the independence of a lot of care-receivers. ${ }^{(3)}$

In addition, Japanese conventional houses don't have enough space which large size robots move. Therefore, installed smaller, lighter, and more mobile care-robots are required. ${ }^{(4-5)}$

This study proposes Omni-directional mobile systems with intuitive operation which is sensed centroid of system by any loads to designate moving direction automatically. ${ }^{(6)}$ Thus, our systems is proposed for developing to support independence of care-receivers and to reduce works of carers.

\section{Experimental}

\subsection{Omni-directional mobile system}

Figure 1 shows the apparatus of developed Omni-directional moving system in this study. A pilot board on a disk and drive this systems. Pilot can control the system with intuitive gravity operation. 


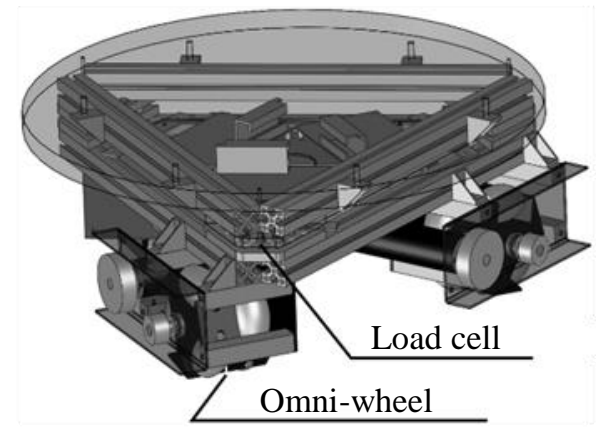

Fig. 1. Apparatus of developed Omni-directional mobile system

The size of the mobile device is 540 (w) $\times 540$ (d) $\times 210$ (t) $\mathrm{mm}$ respectively. Also, the device equips a disc (diameter: $540 \mathrm{~mm}$ ) as standing base which is on double equilateral triangle (side length: $500 \mathrm{~mm}$ ) frames. These double frames operate as a suspension for servo systems (describe later). Between the double triangle frames, three load cells (describe later) are equipped in vertex of triangle. Considering that the load is concentrated at a point system, using a load cell with a load capacity of $1 \mathrm{kN}$ (LMA-A-IKN, KYOWA). It is able to verify that work even the $90 \mathrm{~kg}$ human passengers. Under the triangle frames, three servo system with Omni-wheels are attached to move the systems. The fabricated device has two unique and novel concepts for driving. First, it is very compact for not spacious houses, i.e., the width of this standing base is close to width of a standing human. Second, by such as structures, this system can move all direction in all moving condition. On the basis of the concepts, a cylinder structure (stacking a disk on a triangular frame) was chosen and realized.

Figure 2 shows the operation flow of this system. The movement direction is directed by the movement vector calculated from the difference between the center of the system X-Y coordinates and the centroid X-Y coordinates. Just after the system is started, the load cell is immediately and automatically calibrated. After completing the calibration, the system starts to the standby mode for pilot boarding. When a pilot boards on the machine, the movement vector is calculated via the difference between the center of system X-Y coordinates and the centroid X-Y coordinates. The machine is driven based on the calculated movement vector. When the pilot gets off the board, the system returns to standby mode for pilot boarding again.

\subsection{Servo system specification}

In this study, servo system with DC motors was fabricated. These DC motors (RE40, Maxon) was applied for the actuator. In addition, this system has $3 \mathrm{DC}$ motor to drive at the same time. These DC motors are required 30 [A] for starting power. Table 1 shows the specifications of battery used in this system. This system has two high voltage batteries and one low voltage battery respectively. These types of battery are lithium-polymer battery, they are equipped for minimization of system, i.e., compact and lightweight. ${ }^{(8)}$

Figure 3 shows the overview of the servo system. The rotational speed of the motor in the machine is controlled using the detected value by the load cells. ${ }^{(7)}$

Figure 4 shows the outline of the servo system. The broken line area in Fig.4 shows the high voltage $(24 \mathrm{~V})$ circuit for driving servo systems. This servo system has proportional- derivative (PD) control using the encoder pulses. The encoder pulses are outputted from the rotary encoder which is attached the motor shaft. In the system, one motor and one microcomputer are one pair. The feedback loop is formed by each pairs. The DC motor is

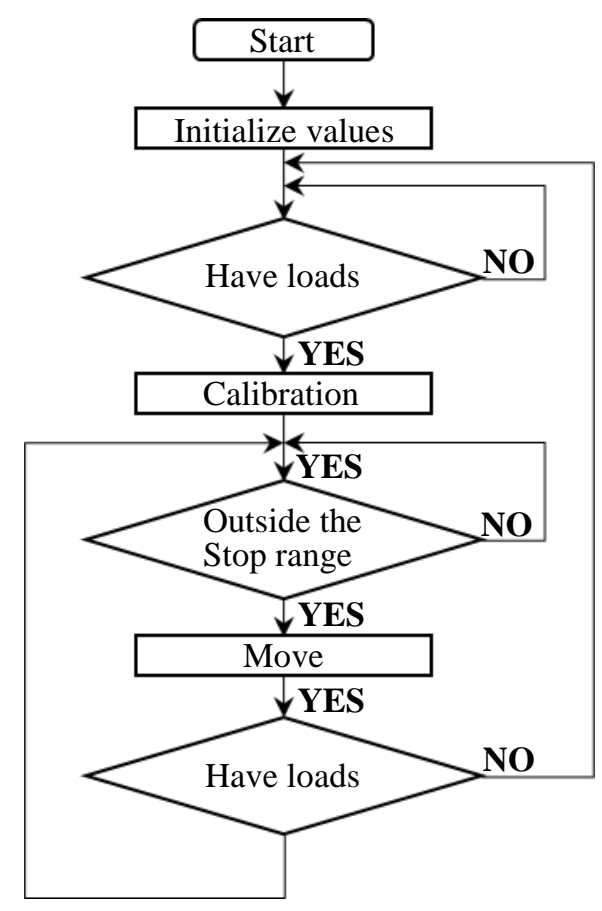

Fig. 2. The operation flow of the servo system

Table 1. Performance of equipped battery

\begin{tabular}{c|cc}
\hline & the high voltage & the low voltage \\
\hline maker & enRoute & IP \\
Model number & enLipoECE21 & THA111850_25CA \\
Rated voltage[V] & 11.1 & 11.1 \\
Rated capacity[mAh] & 1800 & 850 \\
Discharge allowance[C] & 20 & 25 \\
\hline
\end{tabular}




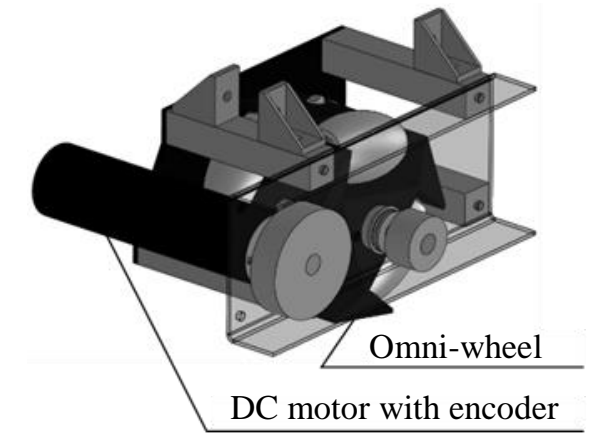

Fig. 3. Overview of the servo system

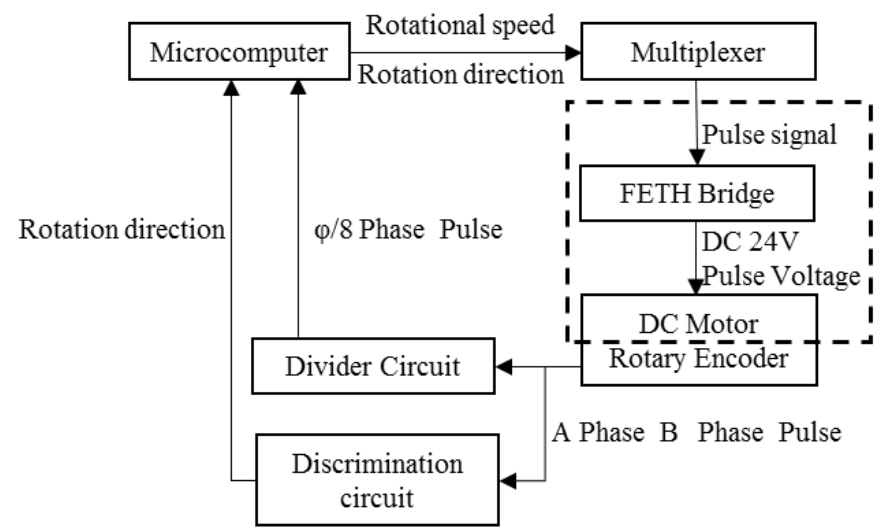

Fig. 4. Outline of the servo system

inputted the rotation direction command and speed command by main microcomputer. These commands are formed 64 steps from 0 to 63 , these steps are formed the rotation signal of 7 bits by the main microcomputer. Finally, DC motors are rotated at the specified speed and direction of rotation. The rotational speed of the motor is controlled using pulse width modulation (PWM).

\subsection{Setting unit of centroid position}

A load cell is designed for setting unit of centroid of the systems. Figure 5 shows the parameters used for calculating the centroid position. The load cell is put on each vertex of equilateral triangle. The analog signal by load cell is converted digital signal $\left(F_{i}\right)$ by the microcomputer.

The total weight of the obtained pilot (or any objects) is $\mathrm{m}$ $\mathrm{kg}$, loads applied on the three load cells are $F_{1}, F_{2}, F_{3}$, and load of centroid is $G$ respectively. These each heights from each side of the equilateral triangle are $h_{1}, h_{2}, h_{3}$ respectively. Equation (1) shows the relationship between $F_{i}$ and $h_{i}$.

$$
m g h_{i}=F i H
$$

Now, $m g$ is the total weight of passengers, it is obtained by total of the loads, $F_{1}, F_{2}, F_{3}$. Equation (1) is modified, and

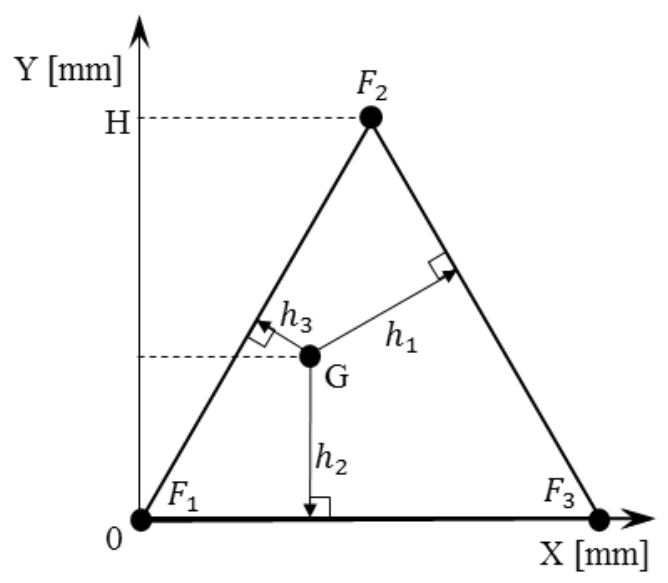

Fig. 5. Diagram of parameter used for calculating the centroid position

$h_{i}$ is calculated by each load and total weight. The equation transformed into Equation (2) is shown.

$$
h i=\frac{F_{i}}{\sum_{k=1}^{3} F_{k}} H
$$

The obtained $h_{i}$ is converted to the $\mathrm{X}-\mathrm{Y}$ coordinates when $F_{l}$ is the origin and sides $F_{1}$ and $F_{3}$ are the $\mathrm{X}$ axis. The $\mathrm{Y}$ coordinate is $h_{2}$ since $h_{2}$ is height from the sides $F_{1}$ and $F_{3}$. Equation (3) shows $X$ coordinate as $s$ and $Y$ coordinate as $t$.

$$
s\left(\begin{array}{l}
1 \\
0
\end{array}\right)+h_{2}\left(\begin{array}{l}
0 \\
1
\end{array}\right)=t\left(\begin{array}{c}
\cos \left(-\frac{\pi}{3}\right) \\
\sin \left(\frac{\pi}{3}\right)
\end{array}\right)+h_{3}\left(\begin{array}{c}
\cos \left(-\frac{\pi}{6}\right) \\
\left(-\frac{\pi}{6}\right)
\end{array}\right)
$$

Therefore, the centroid $\mathrm{G}$ is expressed by the equation (4).

$$
G\left(\sqrt{3 \frac{h_{2}+2 h_{3}}{3}}, h_{2}\right)
$$

\subsection{Investigation of system response}

For investigating the systems' transient response properties, which is measured relationship between system rotating speed driven by DC motor and operation time during 2000 [ms]. In this measurement, systems rotating speed is measured by displacement of rotary encoder per 1 [ms]. The rotation servo system has 64 steps of forward rotation from 0 to 63 and a reverse rotation of 64 steps from 0 to -63 respectively. In this study, the standard maximum motor rotation speed is $8000 \mathrm{rpm}$, one step indicates $8000 / 63=$ $127.0 \mathrm{rpm}$. Here, each commanded rotation speed is 
designated "10" to "60". " $10 "$ is 10 (steps) $\mathrm{x}$

$127.0 \mathrm{rpm}=1270 \mathrm{rpm}$ and "60" is $7619 \mathrm{rpm}$ respectively. Forward rotation mode was investigated in the two conditions. In this study, i.e., under no-loaded condition and under load condition. Measuring under no-loaded condition, the wheel is taken off from the ground and idling. Under loaded condition was measured via a pilot $(60 \mathrm{~kg})$ boarded on the systems.

\subsection{Investigation of accuracy of centroid}

To investigate the direction accuracy by load, accuracy of centroid is measured under two load conditions; $2 \mathrm{~kg}$ PET water bottle as delivering items and $60 \mathrm{~kg}$ human as a pilot. Figure 6 shows the matrix for measuring accuracy. The boarding area, i.e. disk area on triangle frame, is separated 36 pixels per $50 \times 50\left[\mathrm{~mm}^{2}\right]$. The center of each pixel is cross point in the matrix. Each cross points are measurement points. When these loads on the system is put, cylinder (Material: steal, diameter: $42 \mathrm{~mm}$, height: $63 \mathrm{~mm}$ ) is put between loads and the each measurement points. Measurement apparatus is shown in Figure 7. Thus, maximum error distance is the cylinder radius, i.e., $21 \mathrm{~mm}$.

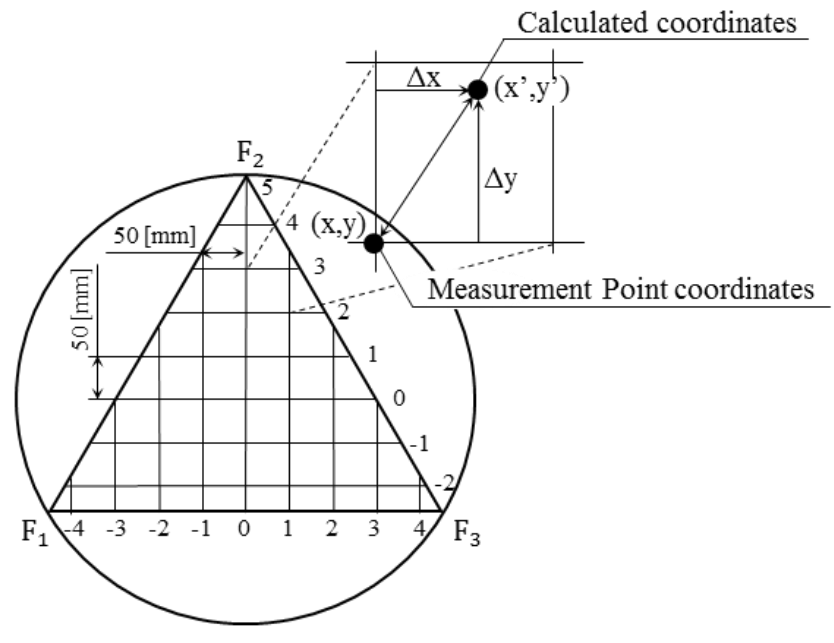

Fig. 6. Matrix for measuring accuracy of cetroid of the system

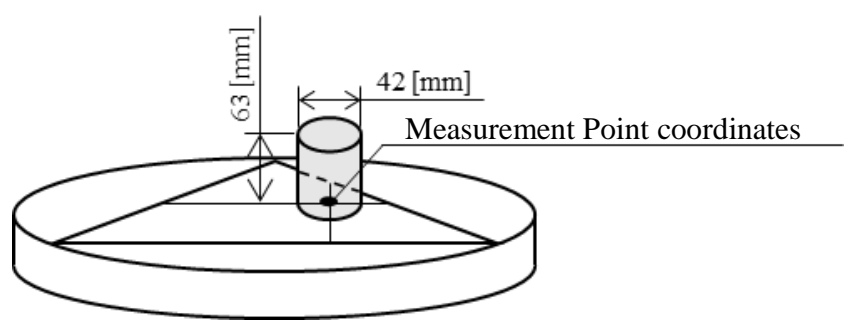

FIg. 7. Measurement apparatus of accuracy of centroid of the system
Now, distance ietween coordinate of measurement point ( $\mathrm{x}$, $y)$ and calculated coordinate of centroid $\left(x^{\prime}, y^{\prime}\right)$ is defir $d$ as $e_{w}$, error distance. Small $e_{w}$ means close to accurate centroid position.

\section{Results and Discussion}

Figure 8 shows the transient response characteristics of the servo system with no-load. Overshoot of rotating are observed in all rotating conditions except for command "60" mode. At the speed-command from "10" to "50", the peaks of the overshoot are observed at almost same timing. After the overshoot and chattering, rotating speeds are converged. The converged speeds are close to each maximum commanded speed in each commands respectively. However, converging time in higher speed mode are longer than lower speed mode.

Between the speed command "10" to "50" and "60", the profiling the servo system rotating speeds are different. Only in speed command "60", it is not observed over shooting so that the system reaches limit of rotation speed of the servo system. However, the commanded speed in command "60" didn't reach maximum commanded rotating speed, i.e., $7619 \mathrm{rpm}$. This means the system cannot rotate over $7600 \mathrm{rpm}$.

Figure 9 shows the transient response characteristics of the servo system with $60 \mathrm{~kg}$ loaded condition. In this result, the wheel slipping was observed in all speed conditions. This slipping is assumed to be caused by inertia throughout the wheels under moving loaded systems. After reached maximum speed, speed value is observed wiggly vibrating. In higher speed, the vibration is smaller. On the other hands, in command "60", the servo system cannot catch up with maximum commanded speed 7619 rpm, i.e., around 1100 rpm lower than maximum commanded speed. Thus, in both results of no-loaded and loaded conditions, the system can be controlled accurately under command "50", i.e. 6349 rpm.

The wiggly vibrating of profiles is assumed to be originated from Omni-wheels structures. These Omni-wheel have been installed 6 small wheels called "barrels" arranged every 60 degrees. Two "barrels" of them are tilted 30 degrees each other, and made a pair. Thus, the barrels have different curvatures between at the center of pairs and the edges of pairs. This difference of curvatures induced rolling resistance. Moreover, the rolling resistance is varied and depended on the ground shape. Now, under the motor transmitted torque to the servo systems, rolling 


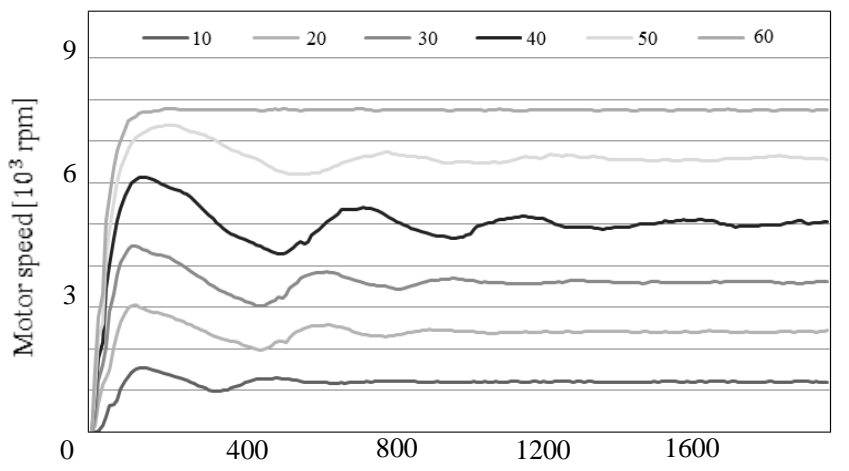

Fig. 8. Transient response characteristics of the servo system with no-load

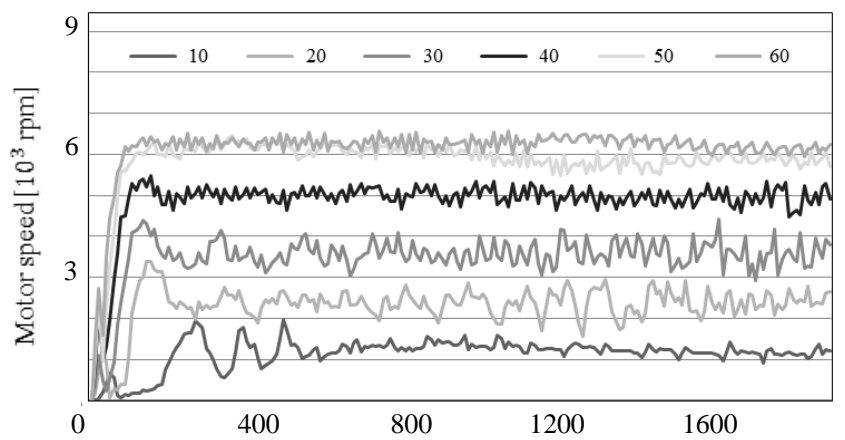

Fig. 9. Transient response characteristics of the servo system with $60 \mathrm{~kg}$ loaded condition

resistance is changed periodically because the motor rotate the shaft also periodically. It means that the angular velocity of the wheel also periodically changes. Thus, the wiggly vibration is generated in this systems.

Figure 10 shows the relationship between rising response time and command number (rotating speed) in no-loaded condition and loaded condition respectively. The rise response time is measured as from rotating speed is $10 \%$ of reaching maximum commanded speed to its $90 \%$. In this results, no-loaded condition profile is not so fluctuated. On the other hands, in loaded condition, rise time is higher than no-loaded condition in all speeds. In addition, in lowest (command "10") and highest (command "60") speed condition, rise time is long like bathtub curve.

Figure 11 shows the relationship between overshoot ratio and command number (rotating speed) in no-loaded condition and loaded condition respectively. Over shoot ratio is calculated by maximum rotating speed per maximum commanded speed. In the no-load condition, it is not observed strong dependence on rotating speed like rise time. However, in loaded condition, the profile is monotonically decreasing in increasing commanded number.

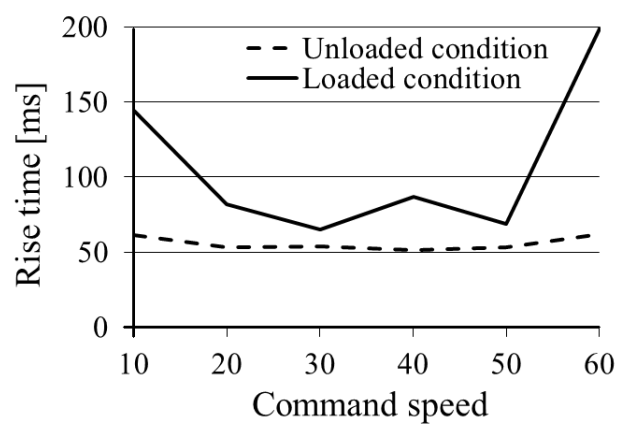

Fig. 10. The relationship between rising response time and command number in no-loaded condition and loaded condition

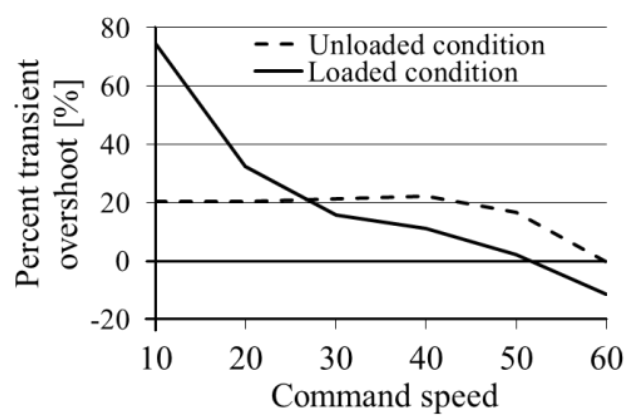

Fig. 11. The relationship between overshoot ratio and command number (rotating speed) in no-loaded condition and condition

On the basis of these analysis, the rotating system cannot control too low or too high rotating speed condition. Especially, over command "60" and 60 heavy loaded condition. It is concluded command "50" (6349 rpm) is the upper limit, command "20" (2540 rpm) is the lower limit in operating this system. In addition, the systems can respond under 100 [ms] in this range of speed, it is enough to apply for real care-robots.

Figure 12 shows the error distance of each pixels on cross point of the matrix (Fig.5) in two difference condition. The $\mathrm{x}$-axis is shown as vertical axis and the $\mathrm{y}$-axis is shown as horizontal axis. The shaded pixels are corresponding to immeasurable area because these positions are too close to Omni-wheels to measure accurately.

In the results of $2 \mathrm{~kg}$ load, all error distance of pixels are under $21 \mathrm{~mm}$. Therefore, the system can detect accurate centroid position loaded light weight subject as $2 \mathrm{~kg}$. On the other hands, in the results of $60 \mathrm{~kg}$, some pixels are observed over $21 \mathrm{~mm}$, i.e. generated measurable error distance. The error distances have a tendency which the measuring point is far from center of disk is larger than near 


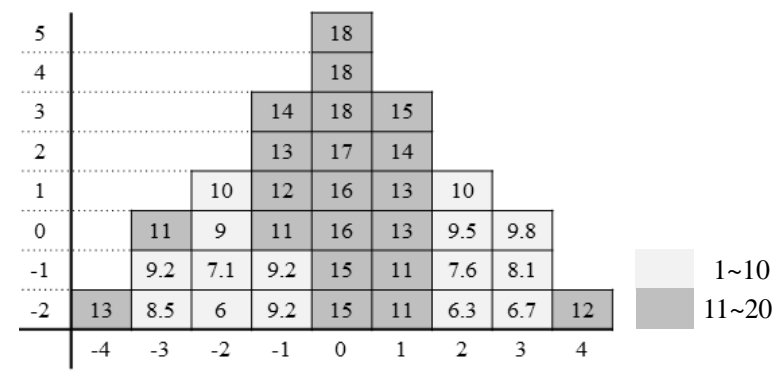

(a) $\operatorname{Load} 2 \mathrm{~kg}$

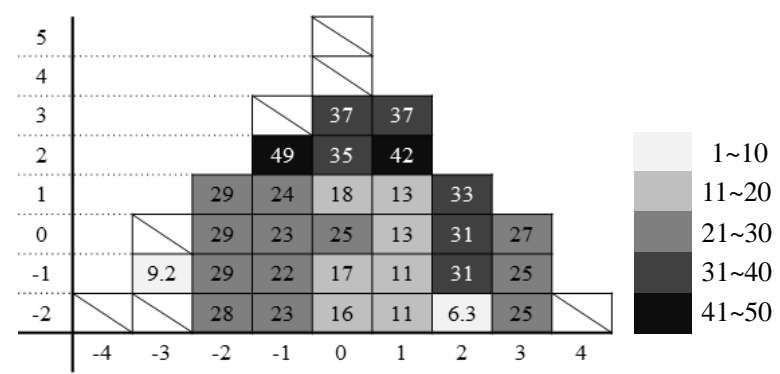

(b) Load $60 \mathrm{~kg}$

Fig. 12. The error distance of each pixels on boarding disk in two difference load condition

center's one. This is assumed to be originated from tilting system by heavy weight like human body. However, according to even maximum value is $49 \mathrm{~mm}$, these value are not so large to apply for practical devices.

\section{Conclusion}

This study proposed a novel and unique intuitive movement system which is installed Omni-wheels with load cells for care-robots. In this systems, pilot or subjects can control intuitively with difference of centroid position.

The fabricated system was investigated via transient response characteristics of the servo system with no-load or $60 \mathrm{~kg}$ loaded condition respectively. The system can move under $60 \mathrm{~kg}$ load. To control the rotating system, commanded rotating speed range from 2540 to $6349 \mathrm{rpm}$ is suitable in responding time and stable rotating speed. By the measuring error distance of centroid, this systems has good accuracy of sensing centroid.

According to these results, the Omni-directional mobile systems with intuitive operation is possible to be expected, novel and unique technology for developing practical care-robots.

\section{References}

(1) Tomoe Nogawa : "QOL of Care and welfare (in Japanese) ", Chuohoki Publishing, pp. 25-28, 2005

(2) Satoshi Hashino : "Daily Life Support Robot (in Japanese)", Journal of The Robotics Society of Japan, Vol. 14, No. 5, pp. 614-618, 1996

(3) Yamaguchi Masaki and Kazuki Takeda and Mitsuru Murakami : "Human Science and Assistive Technology (In Japanese)", CORONA Publishing, pp. 51-54, 2007

(4) Shigenobu Fujiwara and Sei Kitano and Hideki Yamashita and Yuji Maeda and Hideo Fukunaga : "Operation Improvement of The Omni-directional Power Assisted Cart (in Japanese)", Matsushita Electric Works technical report, pp. 28, 2002

(5) Makiko Sasaki and Naoto and Yanagihara and Osamu Matsumoto and Kiyoshi Komoriya : "Light Weight Personal Vehicle Operated by the Movement of Rider's Centroid (in Japanese)", Journal of The Robotics Society of Japan, Vol. 24, No. 4, pp. 106, 2006

(6) Kan Yoneda and Hisashi Osumi and Takashi Tsubouchi : "Robots creative design (In Japanese)", KODANSHA, pp. 22-27, 2005

(7) Kinji Tanikoshi: "Practical know-how of DC motor (In Japanese)", CQ Publishing, pp. 192-194, 2000

(8) Kensuke Okoshi and Tsuyoshi Michinobu and Kiyotaka Shigehara : "Rechargeable Batteries and Energy Issues Focusing Lithium Batteries in particular (in Japanese)", The Chemical Society of Japan, Vol.56, No.3, pp.110-113, 2008 